\title{
Near-Infrared Fluorescence Endoscopy
}

National Cancer Institute

\section{Source}

National Cancer Institute. Near-Infrared Fluorescence Endoscopy. NCI Thesaurus. Code C122415.

The use of near-infrared light (700-1000 nm) for biomedical imaging with an endoscope. 